\title{
Radiation from multi-GeV electrons and positrons in periodically bent silicon crystal
}

\author{
Victor G. Bezchastnov ${ }^{1} \ddagger$, Andrei V. $\mathrm{Korol}^{2} \xi_{\xi}$ and \\ Andrey V. Solov'yov ${ }^{2} \amalg$ \\ ${ }^{1}$ Institute for Nuclear Physics, Johannes Gutenberg University, \\ Johann-Joachim-Becher-Weg 45, D-55128 Mainz, Germany \\ ${ }^{2}$ MBN Research Center, Department of Physics, Goethe University, \\ Altenhöferallee 3, D-60438 Frankfurt am Main, Germany
}

\begin{abstract}
A periodically bent Si crystal is shown to efficiently serve for producing highly monochromatic radiation in a gamma-ray energy spectral range. A short-period small-amplitude bending yields narrow undulator-type spectral peaks in radiation from multi-GeV electrons and positrons channeling through the crystal. Benchmark theoretical results on the undulator are obtained by simulations of the channeling with a full atomistic approach to the projectile-crystal interactions over the macroscopic propagation distances. The simulations are facilitated by employing the MBN Explorer package for molecular dynamics calculations on the meso- bio- and nano-scales. The radiation from the ultra-relativistic channeling projectiles is computed within the quasi-classical formalism. The effects due to the quantum recoil are shown to be significantly prominent in the gamma-ray undulator radiation.
\end{abstract}

PACS numbers: 61.85.+p,41.60.-m,41.75.Fr,02.70.Uu

¥ On leave from A.F. Ioffe Physical-Technical Institute, Politechnicheskaya Str. 26, 194021 St. Petersburg, Russia and St. Petersburg State Polytechnical University, Politechnicheskaya 29, 195251 St. Petersburg, Russia

$\S$ Permanent address: St. Petersburg State Maritime University, Leninsky ave. 101, 198262 St. Petersburg, Russia

|| On leave from A.F. Ioffe Physical-Technical Institute, Politechnicheskaya Str. 26, 194021 St. Petersburg, Russia 
The phenomena of channeling of relativistic charged particles through the crystalline structures remain in focus of challenging studies, both theoretical and experimental ones, for a number of years (see, e.g., the recent monograph [1] and the references therein). In the channeling mode, the particles move along crystallographic planes or axes such that the trajectories exhibit bound oscillations. Out of this mode, the motion is entirely unbound. The two types of motion are very contrast in properties, and so are the radiations produced by these motions. The radiation from channeling particles possesses distinct spectral features signifying bound oscillations in the trajectories, in contrast to a broad-spectrum incoherent bremsstrahlung radiation from the nonchanneling projectiles.

A variety of conditions have been considered in theoretical simulations on channeling dynamics and radiation, to be mentioned among the many are the recent studies [2, 3, 4, 5, 6]. Most widely addressed is the planar channeling in straight crystals. Here, the bound-type motions of the projectiles are the channeling oscillations in the direction transverse to the planes. The corresponding frequencies $\Omega_{\mathrm{ch}}$ are determined by the crystalline inter-planar potentials and by the projectile energies. In periodically bent crystals, the transverse motions of the projectiles acquire additional undulator oscillations. For relativistic particles, the velocities of motion along the channel are close to the light speed $c$, and the undulator oscillation frequency is $\Omega_{\mathrm{u}}=2 \pi c / \lambda_{\mathrm{u}}$, where $\lambda_{\mathrm{u}}$ is the bending period of the crystal.

The oscillations in motion yield the spectral lines in the radiation emitted by the projectiles. For the ultra-relativistic projectiles, the radiation emerges in a narrow domain of the directions close to the direction of the mean velocity, and the line positions scale according to the second power of the Lorentz-factor $\gamma$ of the projectiles [7]. Possibility to tune these positions by varying the bending period and the projectile energies is an attractive property of the crystalline undulator (CU), a device whose concept was formulated some time ago [8, 9]. Theoretically, it has been rationalized that a CU can produce the monochromatic radiation of sub-MeV to $\mathrm{MeV}$ energies, and different schemes for CUs have been proposed for experiments. An evident experimental manifestation of the CU radiation has yet to happen, and further theoretical studies are important for supporting the experiments.

Since introducing the concept of CU, major theoretical studies on stability of the undulator motions as well as the calculations of the emerging radiation have been concerning the regimes where the particles follow the periodically bent crystalline shapes. For such motions, the undulator oscillation frequencies are smaller than the frequencies of the channeling oscillations, and hence the undulator lines appear in the spectra at the energies below the energies of the channeling lines. Yet another regime, with the opposite relation $\Omega_{\mathrm{u}}>\Omega_{\mathrm{ch}}$ between the oscillation frequencies, has recently been shown [5] possible. There, the particles do not follow the bent profiles and the channeling resembles that in the straight crystal. The latter regime can be achieved in a short-period small-amplitude $\mathrm{CU}$ where the bending period $\lambda_{\mathrm{u}}$ is shorter than the period of channeling oscillations in the straight crystal whereas the bending amplitude 
$a$ is smaller than the inter-planner distance $d$.

The newly proposed short-period small-amplitude CU is a novel one and certainly requires additional theoretical verifications in order to be put forward for the experiments. The simulations reported in Ref. [5] are for the $855 \mathrm{MeV}$ electrons and positrons and come along with the conclusion that the effects of bending are not seen in the trajectories but displayed in the radiation spectra. Such a puzzling outcome has to be verified in order to elucidate the physics of the undulator radiation. This has motivated us to investigate the subject by the theoretical methods more advanced and accurate that those employed in the studies [5].

It is also demanding to study impact of the short-period small-amplitude bending on the electrons and positrons with energies higher than the value $855 \mathrm{MeV}$ considered in Ref. [5]. Current experiments with CUs made of $\mathrm{Si}(110)$ extend to the multi$\mathrm{GeV}$ projectile energies, in particular to the value of $10 \mathrm{GeV}$ provided by the SLAC experimental facility [10]. With increasing the beam energy, the undulator radiation, if produced, appears in a harder spectral domain and becomes more distinct in energies from a much softer channeling radiation. The increasing energy separation between the channeling and undulator lines is very attractive for the experimental detection of the undulator effect and its applications. We therefore focus on the $10 \mathrm{GeV}$ projectiles, for the set of CU parameters considered in [5].

To address the essence of undulator radiation it is imperative to study whether the projectiles acquire the short-period bound oscillations when passing through the CU. Giving the positive answer leaves no puzzle in understanding the spectral lines in $\mathrm{CU}$ radiation. For the multi-GeV particles and the bending periods of hundreds of nanometers, these lines appear at gamma-ray energies only a few times smaller than the energy of the projectiles. Here, the quantum effects in the $\mathrm{CU}$ radiation turn out to be important, in particular these due to the quantum recoil, which were neglected in [5] for the sub-GeV projectiles. Along with providing reliable simulations on the $\mathrm{CU}$ radiation, it is demanding to uncover the significance of the recoil in the spectra, the issue which to the best of our knowledge has yet not been quantitatively examined.

To simulate the channeling dynamics, we employ, as in the previously reported studies [3, 4], the MBN Explorer package [11, 12. This package is designed for highaccuracy computations on a broad variety of phenomena developing at meso- bio- and nano-scales in space and time. The theoretical approaches to channeling simulations with the MBN Explorer advance beyond the approximations applied in the other studies, in particular with respect to computing the projectile-crystal interaction. The case of multi-GeV projectiles is also an important benchmark to verify the capabilities of the MBN explorer.

To compute the radiation from the channeling motion, we use the quasi-classical formalism developed by Baier et al [13]. Here, the radiative transitions of the projectiles are described in terms of the classical trajectories, which allows for a direct link of the calculations to the results on the channeling dynamics. The quasi-classical formalism neglects the quantization of the projectile's motion, which is a safe approximation for 
the energies of the interest. At the same time, this approach includes the effects due to the quantum recoil important for the photon emission energies $\omega$ being no longer negligible compared to the energies $\varepsilon$ of the projectiles.

The spectral intensities are computed by numerical integrations over the trajectories, as described in detail in Ref. [2]. For the periodic motions, a variety of analytical approximations become possible. In particular, the following relation [1] applies to the positions of the undulator peaks in the radiation along the mean velocity of the projectiles:

$$
\omega_{n}^{\prime}=\frac{\omega_{n}}{1-\left(\omega_{n} / \varepsilon\right)}=\frac{2 \gamma^{2} \Omega_{\mathrm{u}} n}{1+\left(K^{2} / 2\right)}, \quad n=1,2,3, \ldots
$$

The integer $n$ enumerates the fundamental $(n=1)$ peak and the higher $(n>1)$ harmonics, with $\omega_{n}$ and $\omega_{n}^{\prime}$ being the peak positions with account for the quantum recoil and neglecting the recoil, respectively. Due to the recoil, the radiation frequencies become smaller than the frequencies neglecting the recoil, $\omega_{n}<\omega_{n}^{\prime}$. The undulator parameter $K$ results from the coupling between the projectile's motion along the mean velocity and the oscillations in the transverse direction. We use hereafter the energy units for the frequencies and notice that the ratio $\omega_{n} / \varepsilon$ is a quantum parameter. We will refer to Eq. (1) when discussing the results of calculations on the CU radiation.

Next we briefly outline the details of the simulations on the channeling dynamics. As we study the channeling in $\mathrm{Si}(110)$, the (110) planes of a straight crystal define the $x z$-plane for our reference coordinate system. The $z$-axis is assigned to the direction of the incoming projectiles. A care is taken to avoid accidental orientations of the beam along crystallographic axes, as this is also done in the most common experiments in order to exclude the axial channeling. To simulate a bent crystal with the MBN Explorer, the coordinates $x^{\prime}, y^{\prime}, z^{\prime}$ of each lattice node are obtained from the coordinates $x, y, z$ of the same node in a straight crystal according to the relations $x^{\prime}=x, y^{\prime}=y+a \cos \left(2 \pi z / \lambda_{\mathrm{u}}\right)$ and $z^{\prime}=z$. For the short-period small-amplitude CUs of our interest, the values of $\lambda_{\mathrm{u}}$ and $a$ are of the hundreds of nanometers and of a few tens of Angstroms, respectively. In order to account for the thermal vibrations, the lattice structure is additionally transformed by random displacements of the atoms with respect to the equilibrium positions. The displacements obey the normal distribution corresponding to the value $0.075 \AA$ for the amplitude of the vibrations.

To uncover a maximum outcome of a possible experiment, we simulate a zeroemittance beam at the crystalline entrance $z=0$. The incoming particles are directed along the (110) planes of the reference straight crystal, and the Monte-Carlo sampling over the transverse coordinates covers all different entrance points available for the beam to get into the inside of the crystal. With the above initial conditions, the MBN Explorer solves numerically the relativistic equations of motion to determine the projectile's trajectories. In course of the integration, the interaction of the projectiles with the crystalline environment is computed as a multi-center interaction with all those atoms that influence the motion. The latter atoms are dynamically selected within appropriately large simulation box, and the single interactions are described by the 
Moliere potentials. The relevant details can be found in, e.g., Ref. [2]. Here we would like to stress that, the above approach treats the forces experienced by the projectiles in a much more accurate way than the commonly employed mean-field approach or a particular "snap-shot" approximation to the forces employed in the studies [5]. With the MBN Explorer, the robust Moliere interactions are summed up over the macroscopic propagation sizes and the numbers of the atoms. For example, for the propagation length of $10 \mu \mathrm{m}$ in $\mathrm{Si}$, the interactions with approximately $2.5 \times 10^{6}$ atoms are included into the numerical simulations on the dynamics of the projectiles. For each set of $\mathrm{CU}$ parameters, sampling the initial conditions gave rise to more than 3000 trajectories that have been computed.

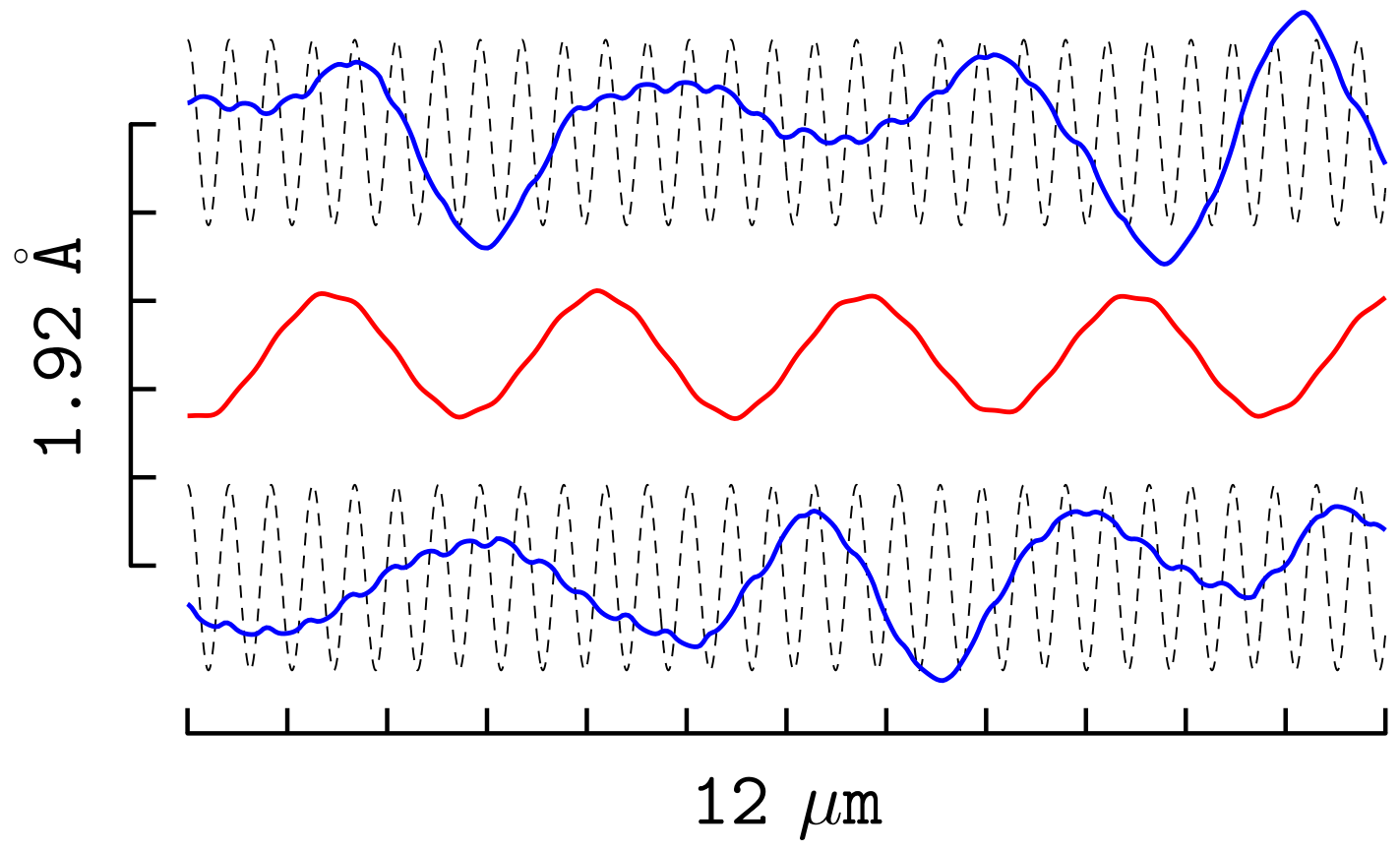

Figure 1. (color online). Effect of the short-period $\left(\lambda_{\mathrm{u}}=400 \mathrm{~nm}\right)$ small-amplitude ( $a=0.4 \AA$ ) bending on channeling of the $855 \mathrm{GeV}$ projectiles in Si. Solid lines are the $12 \mu \mathrm{m}$ long trajectory segments for the channeling motion, whereas the dashed lines are the profiles of the bent crystalline (110) planes. The distance between the mid-lines of the profiles is equal to the inter-planner distance in the straight crystal (1.92 $\AA$ ) and is marked by the vertical scale. The electron and positron trajectories spread through and in between the bent profiles and are colored blue and red, respectively, in the color figure. Clearly seen in the trajectories are short-period modulations resulting from the bent crystalline structure.

First we have simulated propagation of the $855 \mathrm{MeV}$ projectiles in Si CU with $\lambda_{\mathrm{u}}=400 \mathrm{~nm}$ and $a=0.4 \AA$. Opposite to the conclusion of Ref. [5] for this CU and the beam energy, we find the effect of bending to be well displayed in the trajectories of the projectiles. This is demonstrated by Figure 1 showing the typical segments of the channeling motion over the $12 \mu \mathrm{m}$ propagation scale along the bent crystal. The small 
yet well seen oscillations in the trajectories are evident to acquire the undulator period $\lambda_{\mathrm{u}}$ and thus to be responsible for the undulator lines in the radiation spectra.
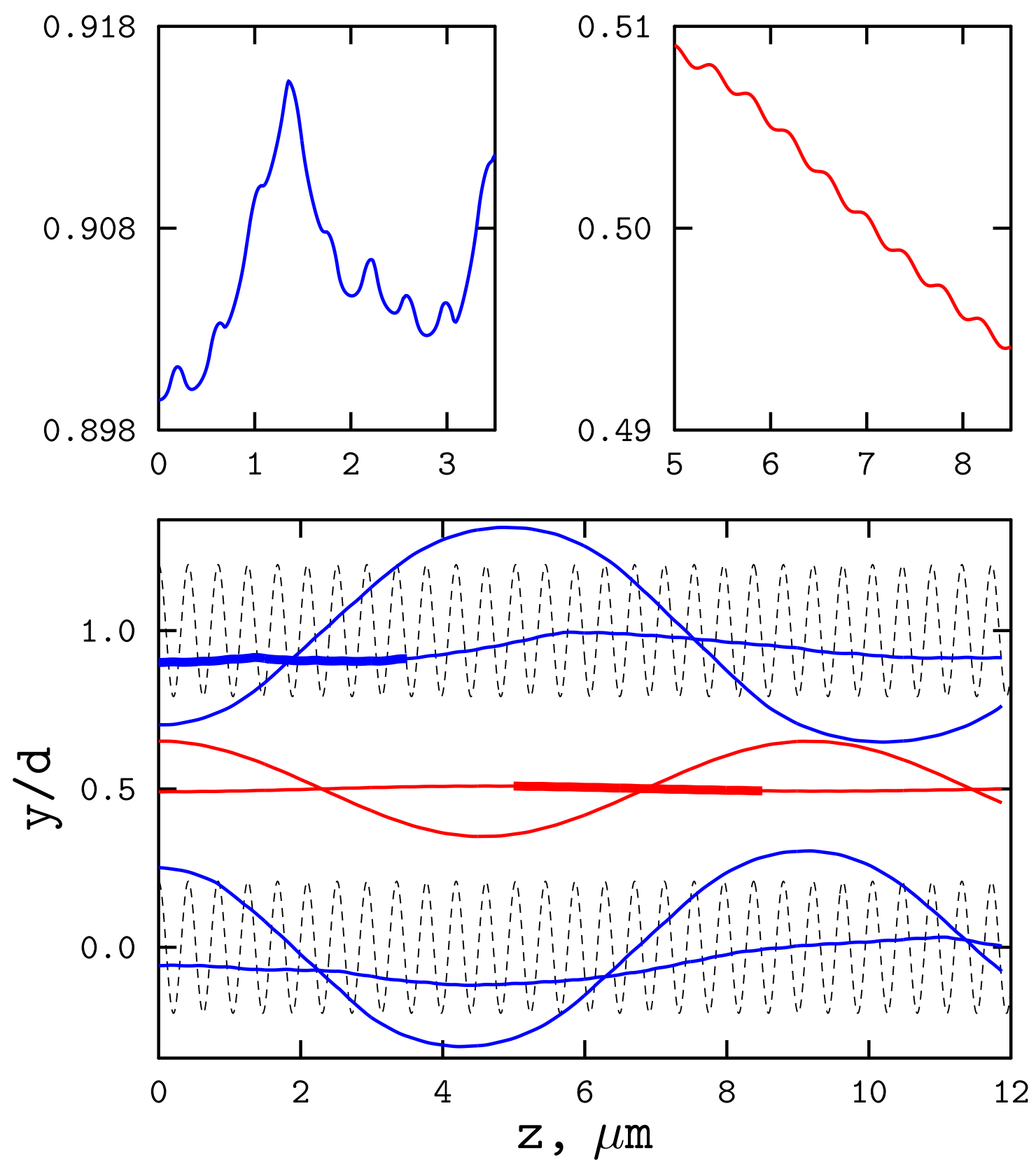

Figure 2. (color online). Channeling of the $10 \mathrm{GeV}$ projectiles through a $12 \mu \mathrm{m}$ thick Si undulator with $\lambda_{\mathrm{u}}=400 \mathrm{~nm}$ and $a=0.4 \AA$. The trajectories and the crystalline profiles are shown by the same types of lines as in Fig. 1. The short-period undulator oscillations are prominent in the trajectories with small mean amplitude of the transverse oscillations - see the separate plots corresponding to the segments displayed by thicker lines.

Next we have studied the channeling and radiation for the multi-CeV particles. 
Having analyzed the simulated trajectories, we can confirm the channeling to indeed occur for the $10 \mathrm{GeV}$ projectiles, both for the electrons and positrons, in the shortperiod small-amplitude CUs. The variety of typical channeling trajectories appears to be distributed between two distinct trajectory types different in mean amplitude of the transverse oscillations. The latter types are shown in Figure 2 and are selected from the simulations for the CU with $\lambda_{\mathrm{u}}=400 \mathrm{~nm}$ and $a=0.4 \AA$. Also shown in the figure are the bent profiles for the (110) planes in Si. The trajectories and profiles are plotted in terms of the transverse coordinate $y$ (in units of the inter-planner distance $d=1.92 \AA$ for the straight $\mathrm{Si}$ crystal) and the longitudinal coordinate $z$ (in $\mu \mathrm{m})$. We remark that our simulations are the 3D ones, and the 2D plots are provided for better visualization of the dynamics of channeling.

The short-period bent crystalline profiles in Figure 2 define the channels guiding the projectiles through the crystal. In the channeling mode, the electron and positron trajectories pass through and in between the bent profiles, respectively. One type of the trajectories studied in the figure are the long-period large-amplitude trajectories. These exhibit two large oscillations along the $12 \mu \mathrm{m}$ pass through the crystal. For the electrons, the oscillation amplitudes slightly exceed the bending amplitude $a$, whereas for the positrons the oscillations get within nearly all the space free from the atoms in between the bent crystalline planes. Another kind of the trajectories are the short-period small-amplitude ones. On the scale of bending amplitude, the corresponding motions are rather close to a translational motion of the free projectiles, though for the electrons in a valuably less extent than for the positrons. Yet on the smaller transverse scale, these trajectories display the oscillations that obviously result from the bent crystalline profiles (see the parts of the trajectories displayed in two separate plots in the figure). The maximum number of the oscillations in a trajectory is given by the ratio $12 \mu \mathrm{m} / \lambda_{\mathrm{u}}$, i.e. is 30 for the $\mathrm{CU}$ with $\lambda_{\mathrm{u}}=400 \mathrm{~nm}$ studied in Figure 2 .

To this end, we focus on the properties of CU radiation by the $10 \mathrm{GeV}$ projectiles. Our concern are the spectral intensities $\mathrm{d}^{2} E(\theta=0) / \mathrm{d} \omega \mathrm{d} \Omega$ calculated as the energy $\mathrm{d}^{2} E$ emitted in the direction of the mean velocity of channeling particles $(\theta=0)$ per photon energy $\mathrm{d} \omega$ and per solid angle $\mathrm{d} \Omega$.

Two kinds of oscillations in the trajectories discussed above contribute differently to the radiation spectra. The long-period oscillations determine the channeling radiation with the peak intensities in the soft energy range. Thought this radiation is similar to the radiation from straight crystal, it is influenced by the bending. In particular, increasing the bending amplitude suppresses the channeling radiation. The latter effect is demonstrated in Figure 3 and is related to decreasing portion of the long-period large-amplitude trajectories in CUs with larger bending amplitudes.

The short-period trajectories contribute to the undulator radiation from the CU. The corresponding spectral lines appear at the gamma-ray energies, as demonstrated in Figure 4. The spectra in this figure are computed for CUs with the amplitude $a=0.4 \AA$ and three periods, $\lambda_{\mathrm{u}}=600 \mathrm{~nm}, \lambda_{\mathrm{u}}=400 \mathrm{~nm}$ and $\lambda_{\mathrm{u}}=200 \mathrm{~nm}$, respectively. Shown by the solid lines are the spectra computed with account for the quantum recoil, whereas 
Radiation from multi-GeV electrons and positrons in periodically bent silicon crystal 8

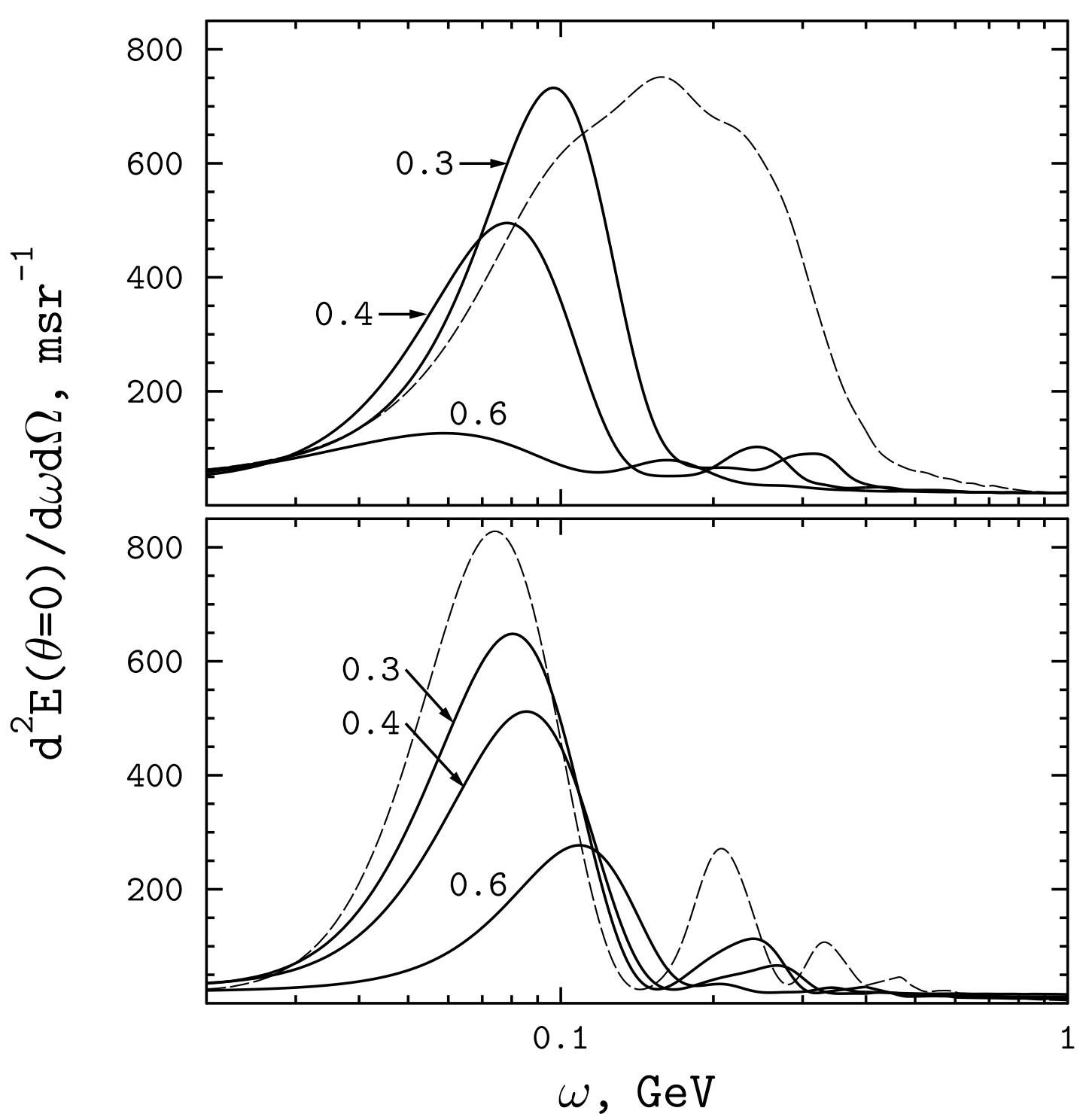

Figure 3. Impact of the bending on the channeling radiation by electrons (upper plot) and positrons (bottom plot). Thin dashed curves are the spectra for the planar channeling in a straight $12 \mu \mathrm{m} \operatorname{Si}(110)$. Thick solid curves are the spectra for the cosine bending profiles of the crystal, with the period $400 \mathrm{~nm}$ and different amplitudes (the amplitude values in $\AA$ are indicated near the curves).

the dashed lines are the results of neglecting the recoil in calculations. An immediate impact of the figure are the striking differences in the positions of the undulator peaks computed with and without accounting for the recoil. The latter is not a surprise as including the recoil yields the fundamental undulator peaks at the energies $1-3 \mathrm{GeV}$ far not negligible compared to the beam energy of $10 \mathrm{GeV}$. These peaks appear at the softer energies, as compared to the peaks computed by employing a pure classical formalism. We also notice that the recoil yields the fundamental and higher undulator 
Radiation from multi-GeV electrons and positrons in periodically bent silicon crystal 9

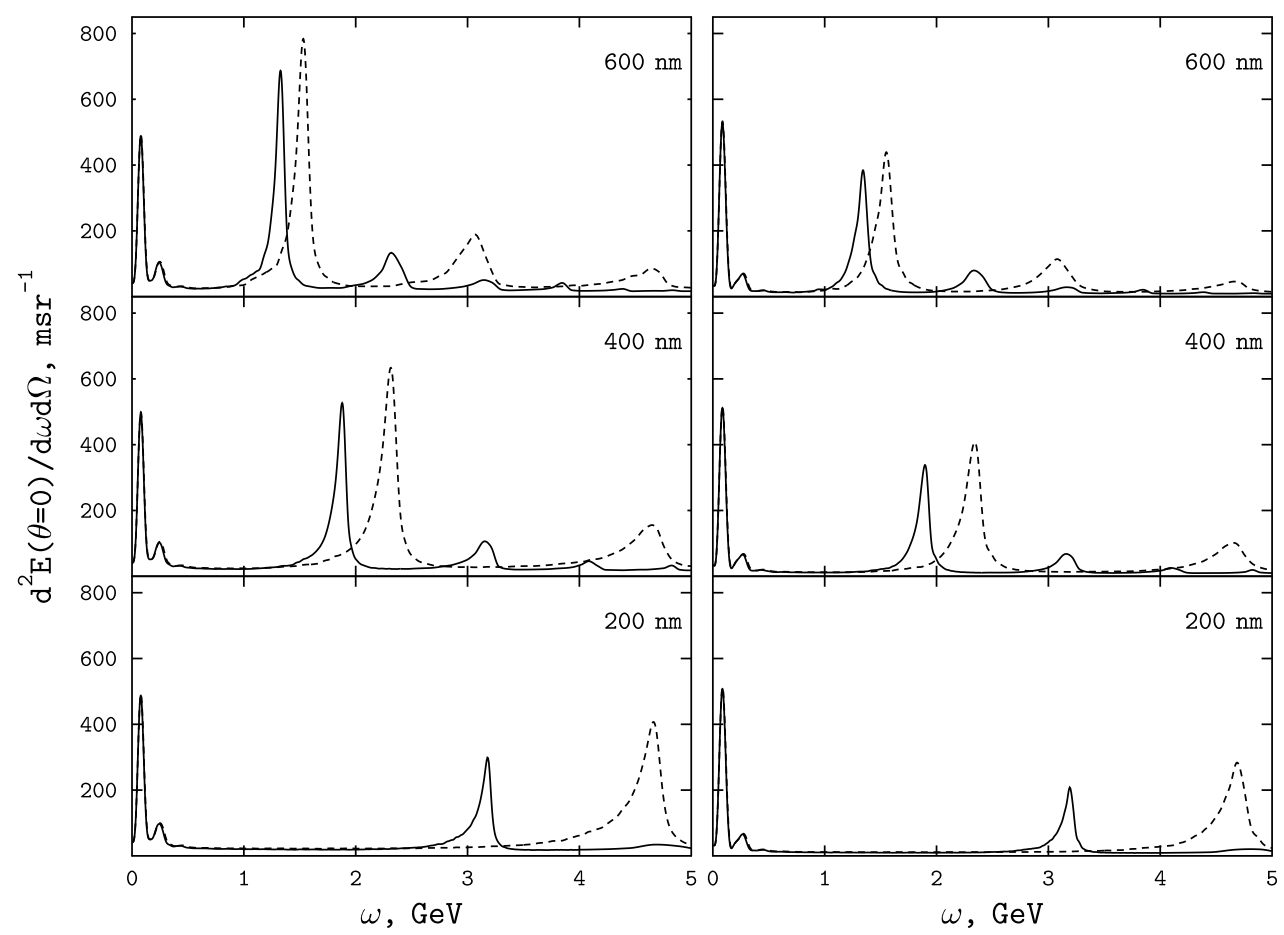

Figure 4. Radiation spectra produced by the projectiles of the energy $\varepsilon=10 \mathrm{GeV}$ propagating through a $12 \mu \mathrm{m}$ thick $\mathrm{Si}$ undulator. The crystalline channels have cosine profiles with the amplitude $0.4 \AA$. Left and right panels show the spectra by electrons and positrons, respectively, for different values of the bending period indicated in the upper right corners of the plots. The dashed-line curves are the spectra computed according to the classical formalism (i.e. assuming $\omega / \varepsilon \rightarrow 0$ and neglecting thereby the quantum recoil).

harmonics to be non-equidistant in spectral locations, in contrast to the equidistant peaks computed within the classical approximation. In contrast to the undulator peaks, the channeling peaks emerge at the energies $0.1-0.3 \mathrm{GeV}$ much smaller than the beam energy. Therefore the channeling parts of the spectra are not influenced by the recoil in any noticeable way.

\begin{tabular}{|c|c|c|cc|c|cc|c|c|}
\cline { 4 - 10 } \multicolumn{2}{c|}{} & \multicolumn{3}{|c|}{ electrons } & \multicolumn{3}{|c|}{ positrons } \\
\hline $\begin{array}{c}\lambda_{\mathrm{u}} \\
\mathrm{nm}\end{array}$ & $\begin{array}{c}\Omega_{u} \\
\mathrm{eV}\end{array}$ & $\begin{array}{c}2 \gamma^{2} \Omega_{\mathrm{u}} \\
\mathrm{GeV}\end{array}$ & $\begin{array}{c}\omega_{1}^{\prime} \\
\mathrm{GeV}\end{array}$ & $\omega_{1}$ & $K$ & \multicolumn{2}{c|}{$\begin{array}{c}\omega_{1}^{\prime} \\
\mathrm{GeV}\end{array}$} & $K$ \\
\hline 600 & 2.06 & 1.58 & 1.53 & 1.33 & 0.26 & 1.55 & 1.34 & 0.20 \\
\hline 400 & 3.09 & 2.38 & 2.31 & 1.88 & 0.23 & 2.34 & 1.90 & 0.18 \\
\hline 200 & 6.18 & 4.75 & 4.66 & 3.18 & 0.20 & 4.69 & 3.19 & 0.16 \\
\hline
\end{tabular}

Table 1. Undulator frequencies for the motion and the spectral lines for the $10 \mathrm{GeV}$ projectiles in CU with the amplitude $a=0.4 \AA$ and different periods $\lambda_{\mathrm{u}}$.

In Table 1 we provide the central frequencies for the undulator peaks. The 
frequencies $\omega_{1}$ and $\omega_{1}^{\prime}$ are determined from the numerical simulations of the spectra including and neglecting the quantum recoil, respectively, and appear to be in a decent agreement with the theoretical result (1). Also included in the table are the values for the undulator parameter $K$ which are deduced from comparing $\omega_{1}$ and $\omega_{1}^{\prime}$ with the values $2 \gamma^{2} \Omega_{\mathrm{u}}$. For all periods of CU studied, the values of $K$ drop far below the value of 1 implying thereby that the bent crystals work indeed as the undulators for the channeling multi-GeV projectiles. With decreasing bending period, the values of $K$ decrease as well being slightly smaller for the positrons than for the electrons.

As the short-period small-amplitude bent Si crystals are being manufactured and the high-quality beams of electrons and positrons with multi-GeV energies are on the present experimental disposal [10], it is demanding to bring these ingredients together for producing and manipulating the monochromatic gamma-ray radiation. Theoretical support of the experiments has to include the effects of the quantum recoil in order to deliver reliable predictions on the radiation spectra. The experimental measurements can also provide the values for the undulator parameter which are valuable for theory of the channeling phenomena.

\section{Acknowledgments}

Financial support due to the European CUTE Project is gratefully acknowledged. We are grateful to H. Backe, W. Lauth and G. Sushko for fruitful discussions, and acknowledge the particular contribution of G. Sushko in developing the MBN Explorer and setting up various aspects of the channeling simulations.

\section{References}

[1] Korol A V, Solov'yov A V and Greiner W 2013 Channeling and Radiation in Periodically Bent Crystals (Springer-Verlag Berlin Heidelberg)

[2] Sushko G B, Bezchastnov V G, Solov'yov I A, Korol A V, Greiner W and Solov'yov A V 2013 J. Comp. Phys. 252404

[3] Sushko G B, Korol A V, Greiner W and Solovyov A V 2013 J. Phys. Conf. Ser. 438012018

[4] Sushko G B, Bezchastnov V G, Korol A V, Greiner W, Solovyov A V, Polozkov R G and Ivanov V K 2013 J. Phys. Conf. Ser. 438012019

[5] Kostyuk A 2013 Phys. Rev. Lett. 110115503

[6] Mazzolari A, Bagli E, Bandiera L, Guidi V, Backe H, Lauth W, Tikhomirov V, Berra A, Lietti D, Prest M, Vallazza E and Salvador D 2014 Phys. Rev. Lett. 112135503

[7] Jackson J D 1999 Classical Electrodynamics 3rd edn (New York: Wiley)

[8] Korol A V, Solov'yov A V and Greiner W 1998 J. Phys. G 24 L45

[9] Korol A V, Solov'yov A V and Greiner W 1999 em Int. J. Mod. Phys. E 849

[10] Uggerhøj U I 2008 Recentr crystalline undulator experimental developments talk at the PECU Meeting, Frankfurt am Main, Germany, 18 Jan. 2008 (unpublished)

[11] Solov'yov I A, Yakubovich A V, Nikolaev P V, Volkovets I and Solov'yov A V 2012 J. Comp. Chem. 332412

[12] http://www.mbnexplorer.com/

[13] Baier V N, Katkov V N and Strakhovenko V M 1988 Electromagnetic Processes at High Energies in Oriented Single Crystals, (Singapore: World Scientific) 